\title{
Thermo-elastic-plastic Model for Numerical Simulation of Fasteners Destruction Under Gasodynamic Impulsive Pressure
}

\author{
Marina Chernobryvko ${ }^{1,2}$, Konstantin Avramov ${ }^{1,2}$, Boris Uspensky ${ }^{1}$, Anatoly Tonkonogenko ${ }^{3}$, and Leopold Kruszka $^{4, *}$ \\ ${ }^{1}$ A.N. Podgorny Institute for Mechanical Engineering Problems, National Academy of Sciences of Ukraine, 61046, Kharkiv, Ukraine \\ ${ }^{2}$ National Technical University "KhPI",61002, Kharkiv, Ukraine \\ ${ }^{3}$ Yangel Yuzhnoye State Design Office, 49008, Dnipro, Ukraine \\ ${ }^{4}$ Military University of Technology, Civil Engineering and Geodesy Faculty, 00-908, Warsaw, Poland
}

\begin{abstract}
Modern rocketry widely employs a method of gasodynamic impulse destruction of bondings which may occur at high variety of temperatures. To design fasteners correctly it is necessary to have the ability to calculate fastener's destruction time at a given pressure. Numerical research is an expedient approach to this problem. A mathematical model of a high-speed deformation and failure in fastening elements of special rocket structures due to gasodynamic wave-impact impulse loading is developed. A technique for numerical analysis of the deformation of fasteners and failure duration is proposed. To perform such analysis a set of factors such as: static stress-strain state due to assembling; thermo-elastic deformation of fasteners due to environment temperature; high-speed dynamical elastic-plastic failure of fastening elements are taken into consideration. The failure model due to the plastic flow considers dynamical material properties. As a criterion of failure maximum plastic deformation is chosen. The technique is implemented for several types of fasteners. Numerical simulation using finite elements method is conducted. The results of the numerical research are well-correlated with experimental data.
\end{abstract}

\section{Introduction}

The tendency to replace numerous experimental studies by numerical modeling for the analysis of the strength and performance of prefabricated structures has been outlined in recent years. For special rocket construction, such modeling is carried out during a design. A distinctive feature of this analysis is the analysis of the time of destruction of the structure. A special rocket construction was considered. Few efforts were made to analyze dynamics and failure of such structures. In the article [1] a method for predicting a fragment velocity which is high or low depending on the fuel-to-mass ratio of the sheath preserved at the time of the failure is presented. Recognizing the limitations of existing methods, the authors devised an analytical approach which properly partitions the available impulse to each major system-mass component. To simulate amplitudes of Mises stresses, finite element model was established by $\mathrm{Qu}$ and Zhan [2]. Engineering models and methods for high-speed dynamics are presented in [3]. Basic equations for thermo-elastic-plastic model are discussed in the article [4]. The results of an experimental and numerical investigation of the destruction of fasteners under gasodynamic impulsive pressure are analyzed in [5].

Thus, the purpose of this paper is:
- development of a technique for analyzing the dynamic strength of a folding construction for a given impulse action,

- determination of the time of destruction of fasteners at high-speed deformation of the construction.

\section{Problem formulation and thermo- elastic-plastic model}

The construction which is shown in Fig 1 is subjected to research.

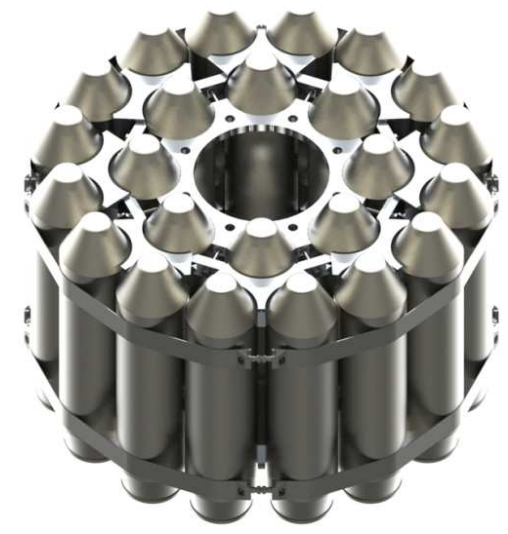

Fig. 1. Special rocket construction.

\footnotetext{
Corresponding author: leopold.kruszka@wat.edu.pl
} 
For the montage of the first stage's payload elements of this construction are used steel bolted joints with aluminum clamping elements. The second stage is bounded to the first one using an elastic tape with a buckle as a regulating element. The schematics of these joints are discussed further in the text.

The force factors acting on the construction are presented in Fig. 2. Curves 1 and 2 show the integral force factors acting on fastening the first and second tiers, respectively. The values of integral force factors were determined experimentally.

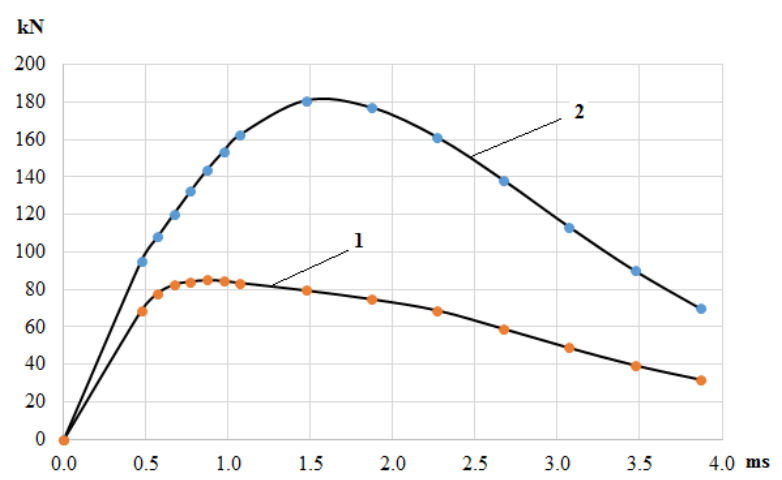

Fig. 2. Force factors acting on the construction.

\subsection{Simulation model}

The geometrical model of special rocket construction for finite-element analysis is presented in Fig. 3. For simulation, the model takes into account the static stress state of the construction.

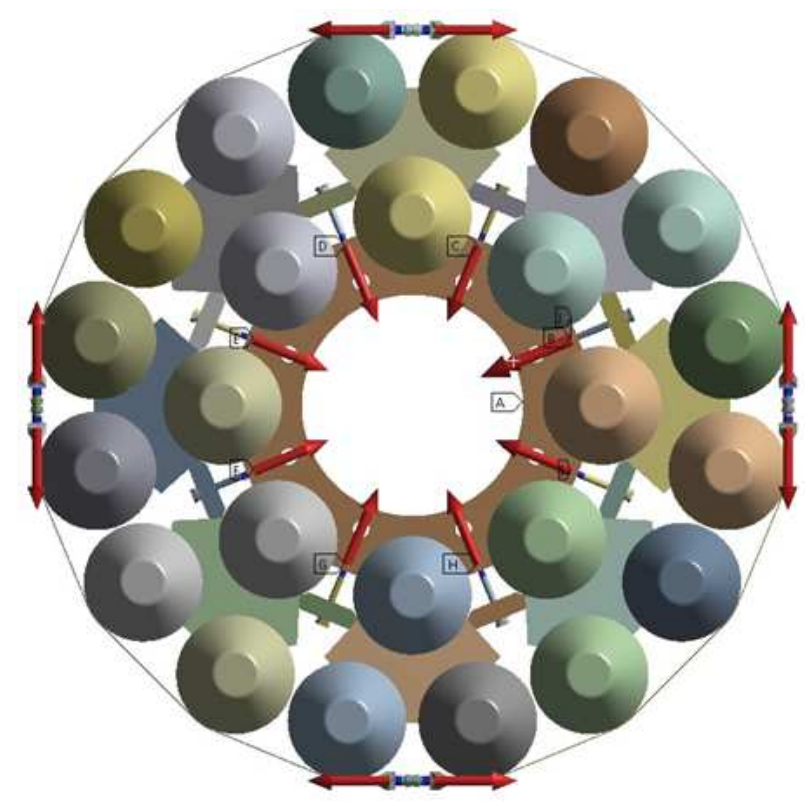

Fig. 3. Geometrical model for the special rocket construction.

Force loads in bolted joints are determined from the conditions of montage for construction. Numerical values of loads are obtained through numerical solution of the static bolt pretension problem using a bolt torque as an input parameter.

\subsection{Thermo-elastic-plastic model}

The analysis of the dynamic deformation of the construction is carried out by the finite element method. However, the area sizes for development of intensive dynamic tensions are limited. Therefore, threedimensional models are used in them.

The equations of motion are presented as follows

$$
(\lambda+\mu) \frac{\partial(d i w v)}{\partial x_{i}}+\mu \nabla^{2} U-3 \alpha K \frac{\partial T}{\partial x_{i}}=p \frac{a^{2} v}{\partial t^{2}}
$$

where $U$ - vector of displacement, $T$ - temperature, $\lambda, \mu-$ Lame parameters, $\rho$ - material density, $\alpha$ - coefficient of thermal expansion, $K$ - module of volume compression.

The set of equations (1) for $i=1,2,3$ is complemented by the initial,

$$
U=0, \frac{\partial v}{\partial t}=V_{0},\left.T\left(x_{i}, t\right)\right|_{t=0}=T_{0},
$$

and the boundary dependences

$$
\left.U\right|_{\Gamma}=F_{1}\left(x_{i}, t\right),\left.\frac{\partial u}{\partial x_{i}}\right|_{\Gamma}=F_{2}\left(x_{i}, t\right),\left.T\left(x_{i}, t\right)\right|_{\Gamma}=T_{0}
$$

The strain tensor is nonlinear.

The stress tensor elements are determined in a dynamic deformation theory

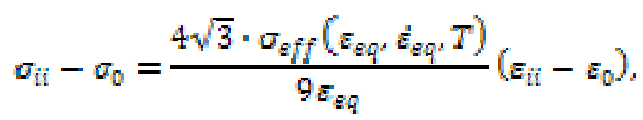

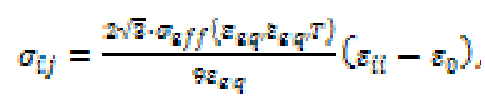

where $\sigma_{0}=\sigma_{i 1} / 3-$ mean value of diagonal element of stress tensor, $\Sigma_{0}=K \sigma_{0}+\alpha T-$ mean value of diagonal element of strain tensor, $s_{e q}$ - equivalent strain, $s_{g q}-$ equivalent strain rate, $\sigma_{i f f}-$ effective stress.

A multiplier $\sigma_{e f f}\left(\varepsilon_{e q}, \varepsilon_{e q}, T\right) / \varepsilon_{e q}$ contains dynamic material properties $\sigma_{a f f}\left(\varepsilon_{a q}, \frac{1}{a q}, T\right)$ :

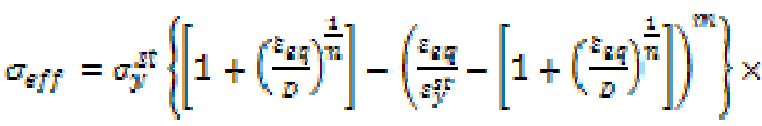

$$
\begin{aligned}
& x\left(1-\frac{T-T_{k}}{T_{p}-T_{k}}\right)^{s}
\end{aligned}
$$

where $\sigma_{y}^{t}-$ the static yield point of materials, $s_{y}^{t}-$ the strain at static yield point, $D, n, m, r$ - experimental parameters of dynamic material properties, $T_{k}-$ temperature which determines material properties, $T_{p}-$ melting temperature of material.

The failure model due to the plastic flow considers dynamical material properties.

The set of equations (1) - (5) allow a full definition of the parameters of the stress-strain state of a structural element under impulsive loading.

By carrying out tests at various strain rates for effective stress, the surface in coordinates: equivalent strain and equivalent strain rate (Fig. 4) should be obtained. 


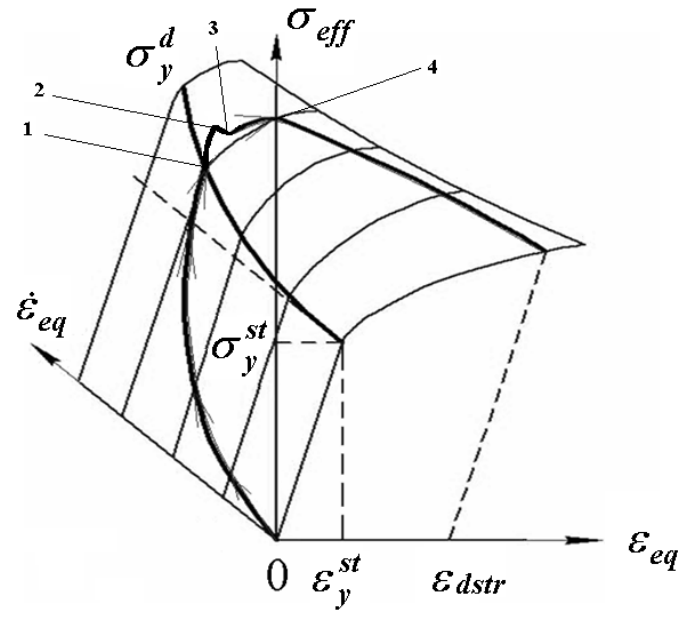

Fig. 4. Dynamic diagram for effective stress.

The constitutive equation with the use of the dynamic deformation theory of plasticity is presented in equation (5). It should also take into account the increase of temperature due to dissipated energy as well as changes in the value of Young's modulus around so called the tooth of strength, i.e. upper and lower yield points (Fig. 5).

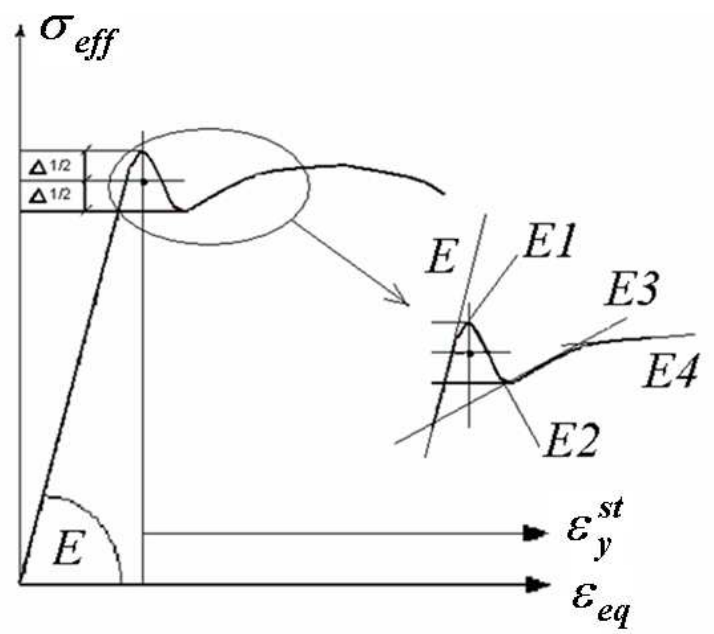

Fig. 5. Stress-strain diagram to determine changes in the values of Young's modulus around upper and lower yield points.

The experimental method allows determining dynamic properties of a number of structural materials under the conditions of variable strain rate.

The proposed model for a local fracture zone by simple simplifications is integrated into a general finite element model for the whole construction.

\subsection{Simplifying the simulation model}

The numerical simulation the destruction of fasteners was carried out to simplify the simulation model. The local models were a part of the global model (Figs 6, 7). The simplified models were loaded with a total load:

1) the load from external force factors,

2) the load derived from the dynamics of all the elements in constructions; these loads are determined numerically.

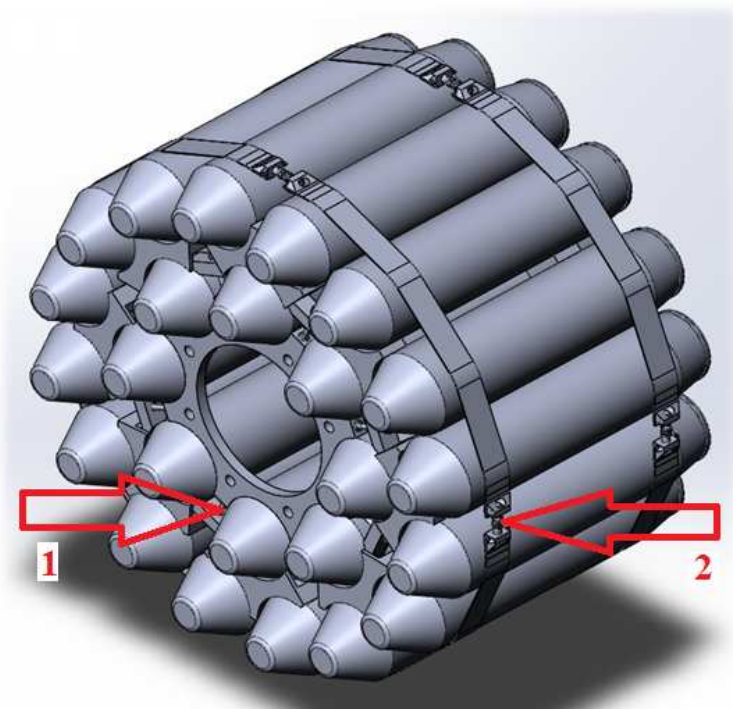

Fig. 6. Two important bolted joints for the special rocket construction in places marked as " 1 " and " 2 ".

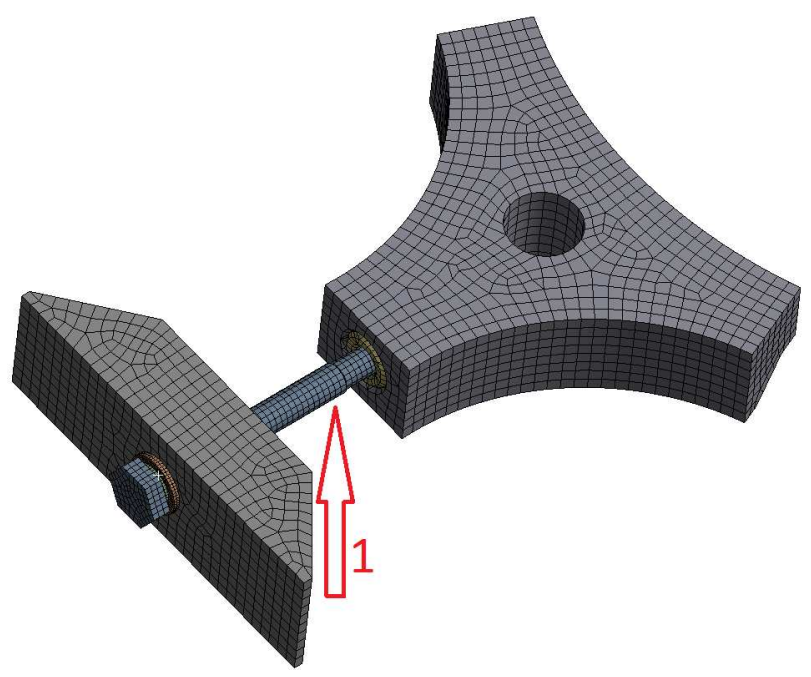

Fig. 7. The bolted joint for the special rocket construction at the place "1" - see Fig. 6.

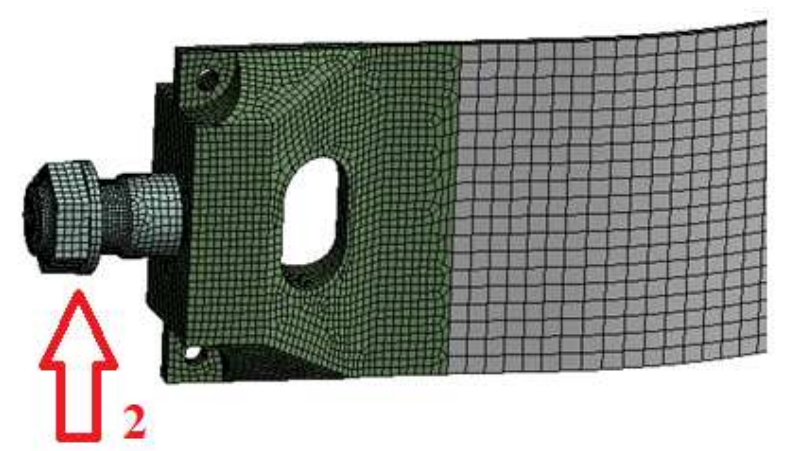

Fig. 8. The bolted joint for the special rocket construction at the place "2" - see Fig. 6.

\section{Numerical simulation}

The numerical simulation was carried out for three types of research: modal analysis, quasi-static analysis and dynamic analysis. 
The physical and mechanical material properties had been provided by Yangel Yuzhnoye State Design Office.

\subsection{Modal analysis}

The first eigenmode of the vibrations is shown in Fig. 9. Fifty forms of oscillations were obtained. For this calculation, the internal fastening was modeled in the construction. To assess fatigue strength, modal analysis was used.

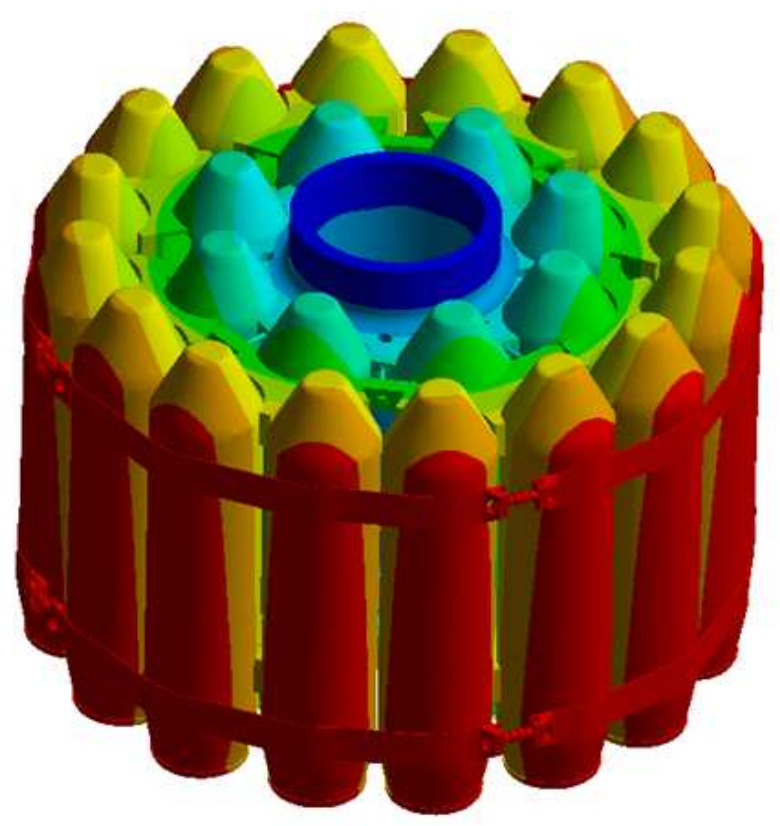

Fig. 9. First eigenmode of the construction vibrations.

\subsection{Static analysis}

Dynamical analysis of the destruction process of such construction is a strongly physically and geometrically nonlinear problem. Its full-scale numerical solution is an extremely complex, costly and hard in terms of convergence task. Thus, reduction of the dynamical analysis object to the destroyed joints is highly desirable. To complete this task, areas of the most dangerous stresses were obtained.

For qualitative analysis and determination of total loads, the static analysis was used. The results of this simulation are shown in Figs $10-15$.

Figures $10-12$ show equivalent stress, equivalent plastic strain and total deformation, respectively, for the first fastening.

Figures $13-15$ show equivalent stress, equivalent plastic strain and total deformation, respectively, for the second fastening.

Static analysis does not include the conclusions for the destruction, but it determines the most dangerous zones of stress and the localization of the plastic deformation.

The time of destruction of the elements of the first and second fastenings is determined in the dynamic simulation of the deformation at the total loading. Such results are shown below.

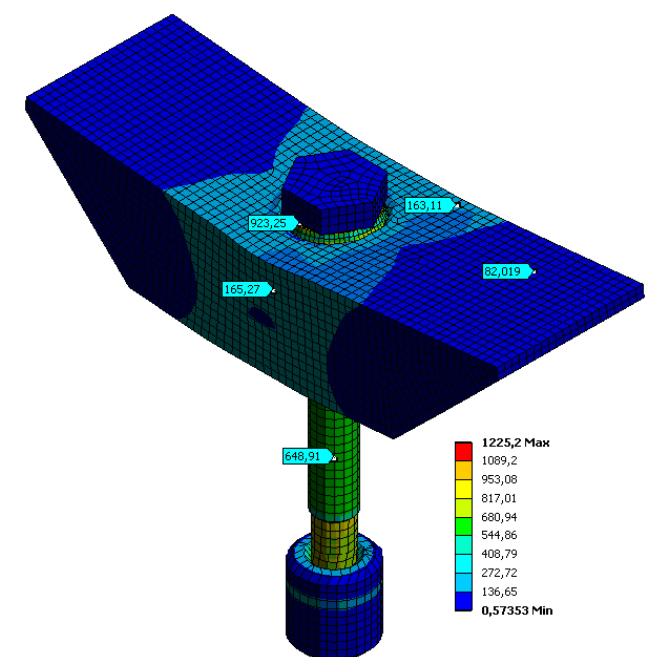

Fig. 10. Distribution of static equivalent stress (unit: MPa) for the first fastening.

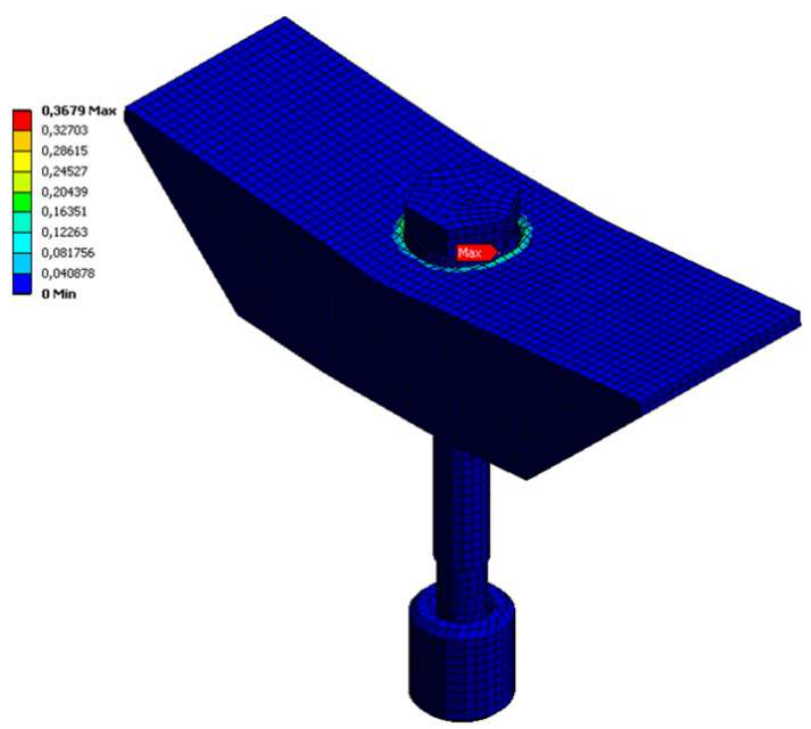

Fig. 11. Distribution of static equivalent strain (unit: $\mathrm{mm} / \mathrm{mm}$ ) for the first fastening.

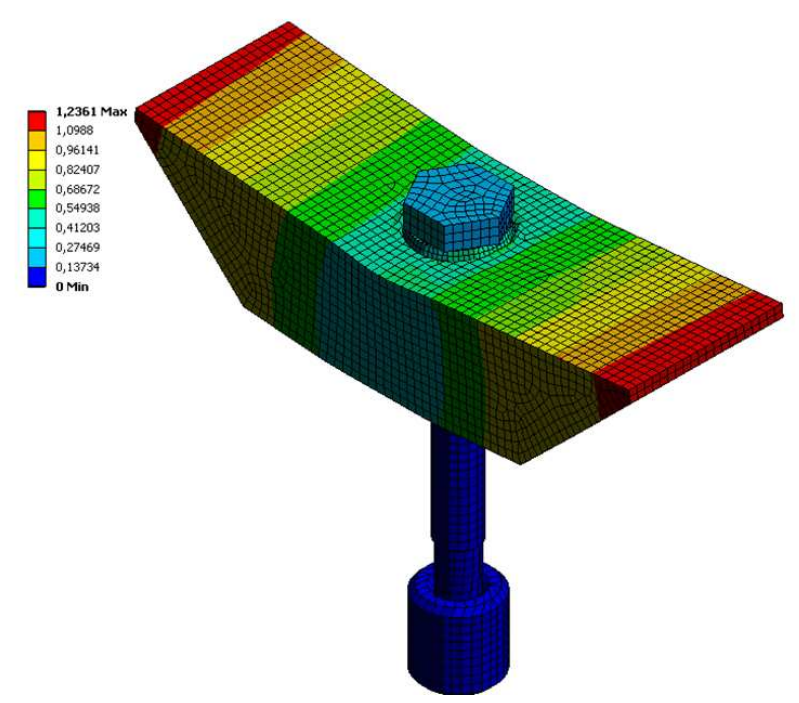

Fig. 12. Distribution of total static deformation (unit: $\mathrm{mm}$ ) the first fastening. 


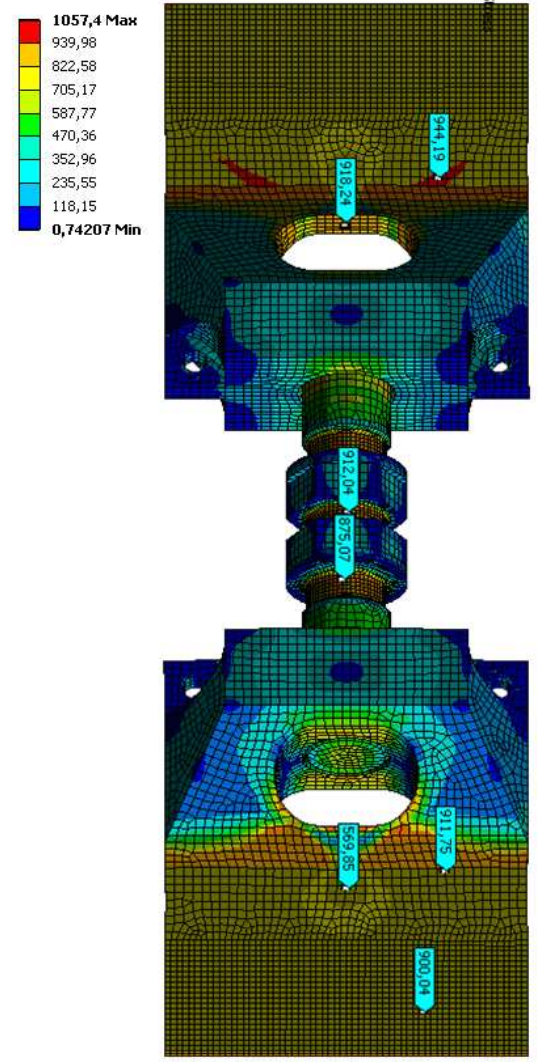

Fig. 13. Distribution of static equivalent stress (unit: MPa) for the second fastening, including localizations of its local maximum values.

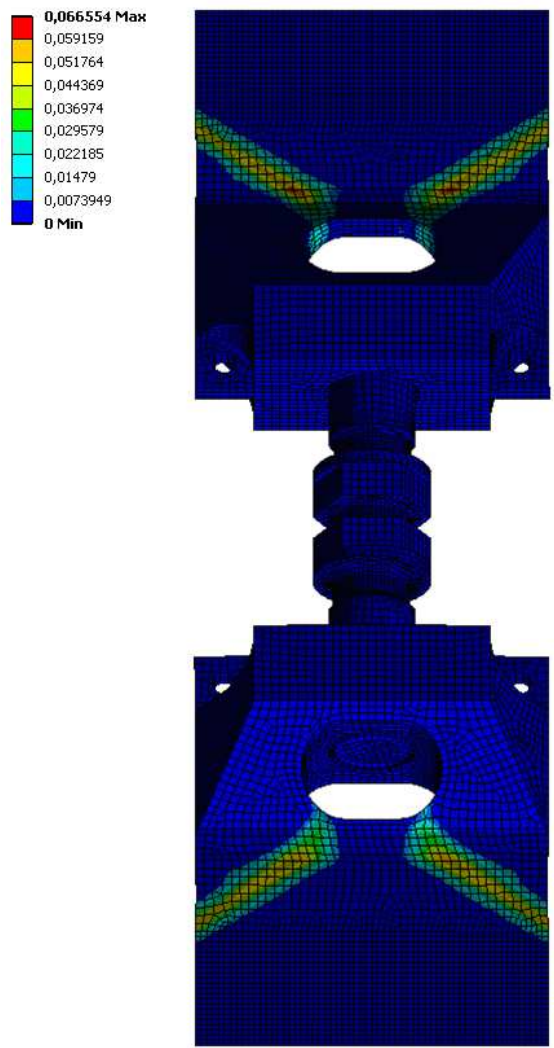

Fig. 14. Distribution of static equivalent strain for the second fastening.

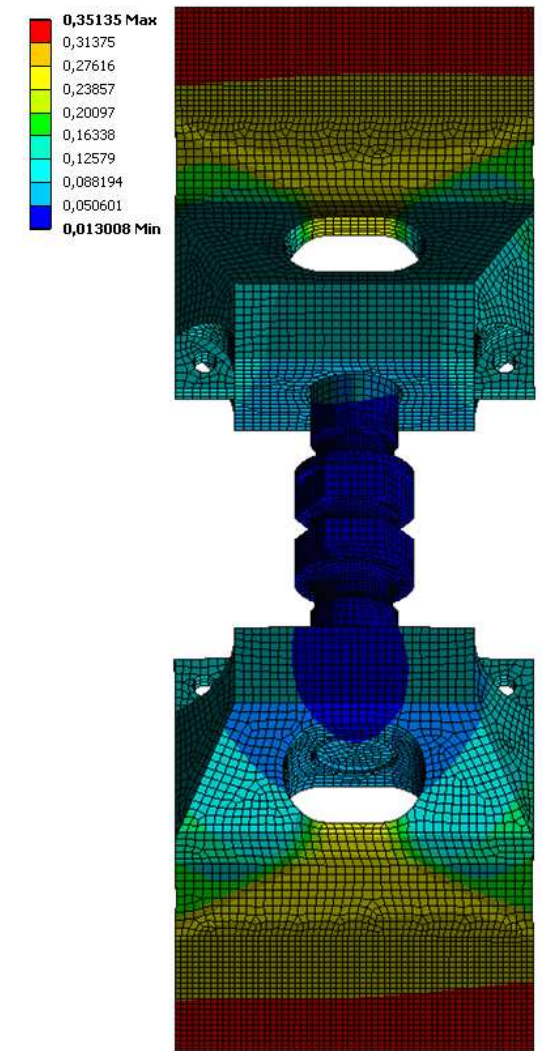

Fig. 15. Distribution of static total deformation (unit: $\mathrm{mm} / \mathrm{mm}$ ) for the second fastening.

\subsection{Dynamic analysis}

The results of dynamic simulation are shown in Figs 16 - 19. The calculation time is synchronized with the loading time. The time of destruction of the first and second fastenings is obtained. The results of the numerical research are well-correlated with experimental data [5].

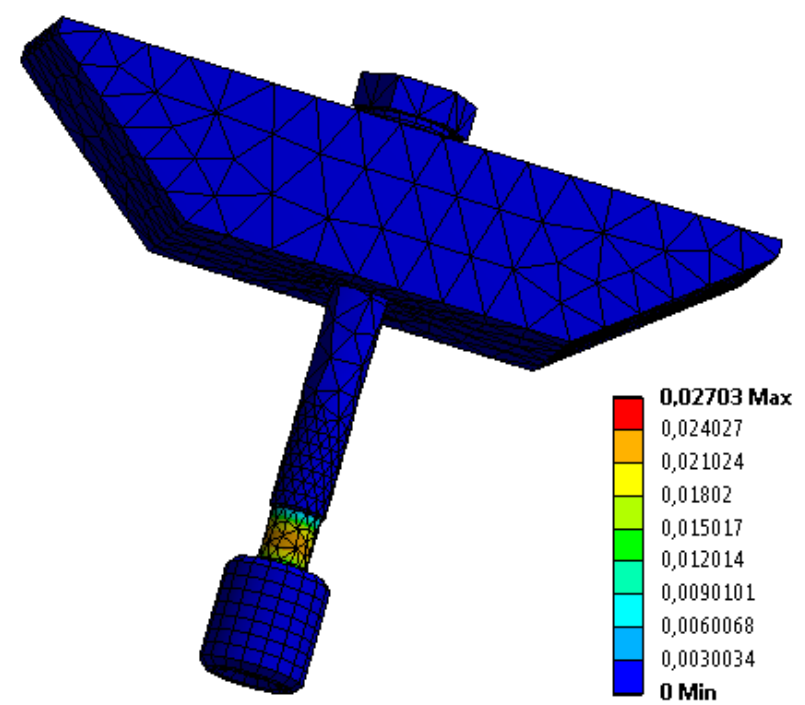

Fig. 16. Distribution of dynamic equivalent strain for the first fastening at calculation time point of $1.3 \mathrm{~ms}$. 


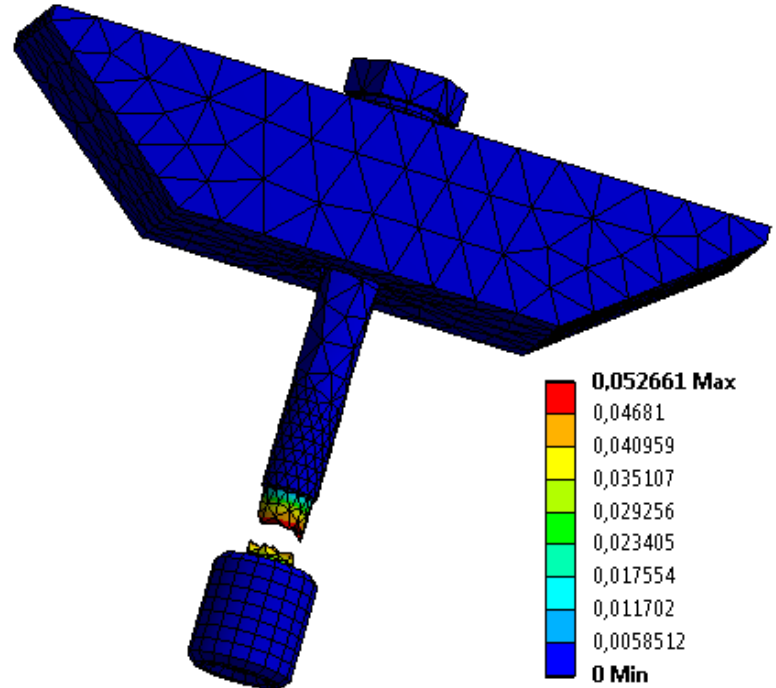

Fig. 17. Distribution of dynamic equivalent strain for the first fastening at calculation time point of $1.4 \mathrm{~ms}$.
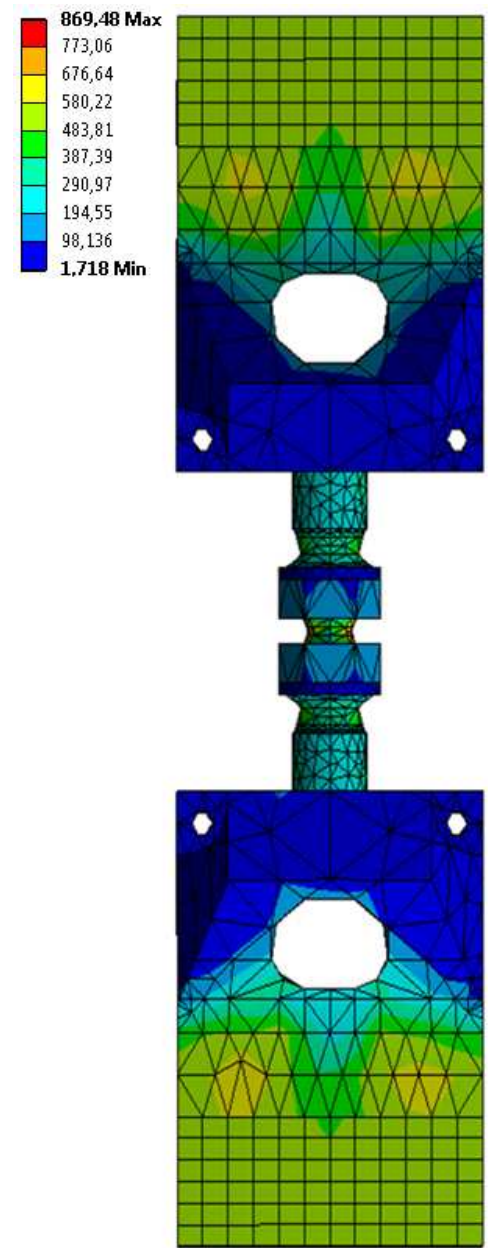

Fig. 18. Distribution of dynamic equivalent stress (unit: MPa) for the first fastening at calculation time point of $1.8 \mathrm{~ms}$.

\section{Summary}

This work presents the mathematical model of a highspeed deformation and failure in the fastening elements of special rocket structures due to gasodynamic waveimpact impulse loading and the technique for numerical analysis of the deformation of fasteners and failure duration. The failure model due to the plastic flow considers dynamical material properties. As a criterion of failure maximum plastic deformation was chosen. The technique was implemented on a few types of fasteners. Numerical simulation using finite elements method was conducted. The results of the numerical research are well-correlated with experimental data.

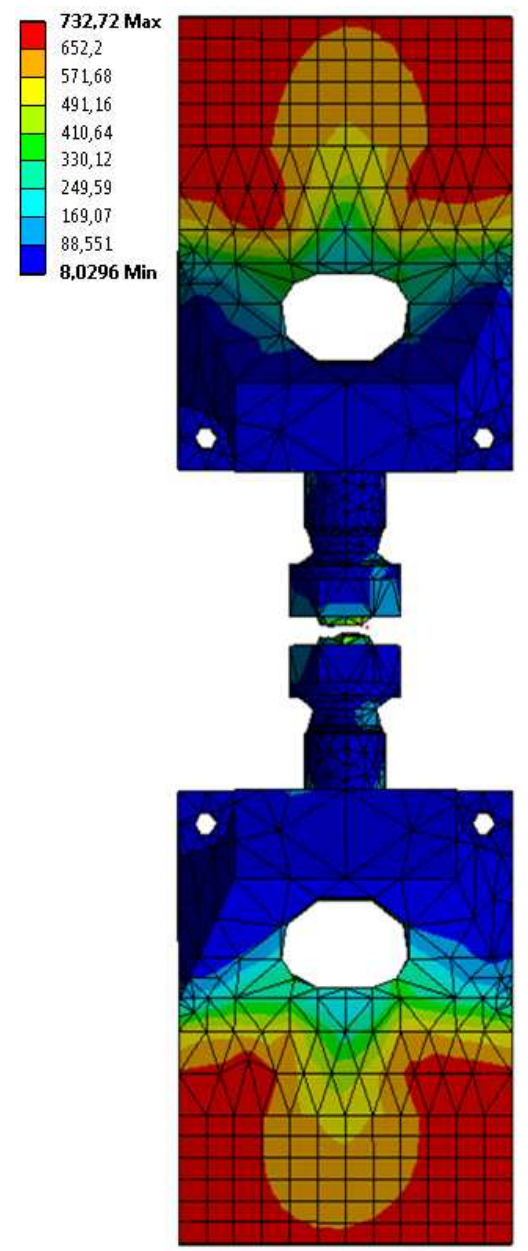

Fig. 19. Distribution dynamic of equivalent stress (unit: MPa) for the second fastening at calculation time point of $1.9 \mathrm{~ms}$.

\section{References}

1. M. Eck, M. Mukunda, Acta Astronaut., 19, 617 (1989)

2. K. Qu, X. Zhang, Propulsion and Power Research, 2, 50 (2013)

3. G. Ben-Dor, A. Dubinsky, T. Elperin, High-Speed Penetration Dynamics: Engineering Models and Methods (World Scientific Publishing, 2013)

4. M. V. Chernobryvko, L. Kruszka, Yu. S. Vorobiev, Applied Mechanics and Materials, 566, 493 (2014)

5. K. Avramov, M. Chernobryvko, G. Martynenko, Working out the methods for simulations the duration of destruction of the fastening elements under impulse loading (Contract No. 149-16 between A.N. Podgorny Institute for Mechanical Engineering Problems, Kharkov, and Yangel Yuzhnoye State Design Office, 2016, in Russian). 